\title{
Non-thermal phase transitions in semiconductors under femtosecond XUV irradiation
}

\author{
Nikita A. Medvedev ${ }^{* a}$, Harald O. Jeschke ${ }^{b}$, Beata Ziaja ${ }^{\mathrm{a}, \mathrm{c}}$ \\ ${ }^{a}$ Center for Free-Electron Laser Science, DESY, D-22607 Hamburg, Germany \\ ${ }^{\mathrm{b}}$ Institut für Theoretische Physik, Goethe-Universität Frankfurt am Main, Max-von-Laue-Strasse 1, 60438 Frankfurt, \\ Germany \\ ${ }^{\mathrm{c}}$ Institute of Nuclear Physics, Polish Academy of Sciences, Radzikowskiego 152, 31-342 Kraków, Poland
}

\begin{abstract}
When a semiconductor or a dielectric is irradiated with ultrashort intense X-ray pulse, several processes occur: first the photoabsorption brings the electron subsystem out of equilibrium, bringing valence or deeper shells electrons into high energy states of the conduction band. Then, secondary electron cascading promotes further electrons of the valence to conduction band increasing their number there. These electrons also influence the atomic motion, modifying the interatomic forces. This process is known as a nonthermal melting. It can turn a material into a new phase state on ultrashort timescales. Recently developed hybrid model for treating all of these processes with different computational tools was reported in [N. Medvedev et al, New J. Phys. 15, 015016 (2013)]. Based on this model, we present here further investigations of nonthermal processes occurring in diamond under irradiation with a FLASH pulse of $10 \mathrm{fs}$ FWHM and $92 \mathrm{eV}$ photon energy. It is shown that the diamond turns into graphite under such irradiation, independently whether constant pressure or constant volume modeling is performed. However, for the latter case, the time of the nonthermal phase transition is longer (few tens of fs for $P=$ const vs few hundreds of fs for $V=$ const) and the damage threshold is slightly higher $(0.69 \mathrm{eV} /$ atom vs $0.74 \mathrm{eV} /$ atom, correspondingly).
\end{abstract}

Keywords: Nonthermal melting, Free-Electron Laser FLASH, Graphitization of diamond

\section{INTRODUCTION}

For last two decades the process of nonthermal melting under femtosecond laser irradiation attracts much attention from both, experimental and theoretical research. It has been studied with femtosecond visible light irradiation of different materials [1-4]. Many interesting phenomena were observed, and a fundamental understanding of the electron-lattice interaction was significantly enhanced. Up to recently, such studies were possible only with visible light sources. But a few years ago, the development of $4^{\text {th }}$ generation of intense free-electron lasers (FELs) allowed addressing femtosecond science with new capacities. These brilliant FEL-sources of extreme ultraviolet and soft X-ray radiation provide short pulses of intensities inaccessible before. A response of irradiated materials to femtosecond X-ray laser pulses has been intensively studied both experimentally [5-15] and theoretically [13-21].

One of the important advantages of femtosecond FELs is that the pulse duration is comparable with fundamental characteristic timescales of the processes in solids: the typical timescales of kinetics of electron subsystem of material, including its nonequilibrium stage. This enables studies of the processes with unprecedented precision (femtosecond temporal scales, down-to-angstrom spatial scales). It is especially important for the nonthermal processes. The diamondto-graphite phase transition occures within a hundred femtoseconds, well before the electron-phonon coupling gives any significant contribution into the lattice heating and phase transition [13, 21, 22]. This means, that the processes involved into the phase transition are strongly interlinked, and must be addressed comprehensively when modeling such effects.

The excitation and relaxation of the femtosecond X-ray irradiated target consists of: (i) electronic excitation by a laser pulse and the subsequent excitations of secondary free-electrons. During this state the electron subsystem is in nonequilibrium state, which typically lasts up to few tens of femtoseconds [17]. (ii) Auger decays of deep atomic shells,

* nikita.medvedev@desy.de

Damage to VUV, EUV, and X-ray Optics IV; and EUV and X-ray Optics: Synergy between Laboratory and Space III, edited by René Hudec, Libor Juha, Saša Bajt, Ladislav Pina, Richard London, Proc. of SPIE Vol. 8777, 877709 . (C) 2013 SPIE · CCC code: 0277-786X/13/\$18 · doi: 10.1117/12.2019123 
if photon energy was sufficient for the excitation. Further relaxation of excited electrons take place the same scales of a few tens of femtoseconds $\left(\sim 10^{-14} \mathrm{~s}\right)[17,23]$. (iii) An energy exchange of electrons with the lattice via modification of interatomic potential which also occurs on the same timescales $[21,22,24]$. Later, electron-phonon coupling mechanism can heat the lattice even further, and macroscopic structural modifications and strain relaxations can take times up to pico- to nano-seconds or even longer [25].

In this report, we apply our recently developed combined code [21] to tackle the nonthermal processes in diamond under femtosecond FEL irradiation. The code consists of different modules: (a) Monte Carlo (MC) part treating nonequilibrium kinetics of high energy electrons after photoabsorption; (b) the module tracing the temperature of a valence and low energy conduction band electrons which are nearly in thermal equilibrium; (c) Molecular Dynamics (MD) tracing the atomic motion of all atoms in our simulation box individually, and (d) the tight binding method for calculations of the transient electron band structure and collective interatomic forces [21]. We briefly describe the methods and approximations used, and apply it at typical FLASH parameters during experiments on irradiation of diamond. We analyze two limiting cases: the constant pressure case in the material which assumes that the material can relax macroscopically (a small simulation box within the large laser spot), and the constant volume case, which relies on assumption of strong constrains from the surrounding material. We demonstrate that the overall behavior is similar in both cases, however, the timescales of the nonthermal phase transition differ.

\section{COMBINED MC-TBMD MODEL}

\subsection{Overview of the model}

Initially photoexcited electrons are brought up into high-energy states of the conduction band. Then they excite secondary electrons via inelastic scattering (secondary impact ionizations) if their energy is sufficient to overcome the band gap of the material. Losing its energy in this process, the electron falls into the Fermi sea of the valence or conduction band electrons, thermalizing with them. It has been demonstrated in a number of works that for the case of XUV or X-ray excited electrons the low-energy electron fraction thermalizes much faster than the whole electron subsystem including the high-energy electron fraction [15-19]. The high-energy electrons, in contrast, remain nonthermalized over a few tens or even hundreds of femtoseconds. Thus, the characteristic shape of the electron distribution function after the intense XUV irradiation [16-19] is referred to as the "bump on hot tail" distribution [20]. That particular shape allows to simplify the model, and treat those two fractions of electrons with different methods. A temperature can be attributed to the low energy electrons, and thus thermodynamic approach can be used for them, while for the high energy electrons nonequilibrium Monte Carlo model should be used, combined together similarly to Ref. [26].

Low energy electrons within the valence and conduction band form the attractive potential for ionic cores in semiconductors and dielectrics. Their distribution function directly enters into the potential energy surface of atoms. Therefore, exciting electrons, we influence the atomic interaction, motion, and ultimately the structure of the material. This forms the driving force for the nonthermal phase transition, when atoms undergo a structural change due to the excited electrons. In turn, the atomic disorder affects the electronic band structure, influencing the distribution of the valence and conduction band electrons. For modeling the lattice dynamics, we apply a molecular dynamics on a potential energy surface evolving at each time step with the tight binding method [21,22]. Such potential energy surface depends on the transient spatial positions of all atoms in the simulation box, and on the distribution function of the electrons. The numerical scheme developed in [21] is presented in Fig.1, and all the details can be found in Ref. [21]. Here we only describe the physical foundation of the simulation methods.

\subsection{Monte Carlo event-by-event simulation of high energy electrons}

We perform a Monte Carlo modeling of event-by-event simulation of individual particles similar to those in Refs. [1618]. In this method we include the photon penetration within the simulation box, secondary cascading of electrons, and Auger-decays of K-shell holes if photon energy was sufficient to excite them. We set photons according to a Gaussian temporal profile with $10 \mathrm{fs}$ as FWHM. The penetrating photons get absorbed by a shell which is chosen according to the relative photon attenuation length for the shell photoabsorption [27]. In case of valence-band photoabsorption, we choose 
a particular energy level randomly among all the valence band levels, which are obtained within the tight binding formalism, see below. We assume that each incoming photon excites only one electron, which is a good approximation for photons at X-ray energies and intensities relevant for solid-solid phase transitions [18].

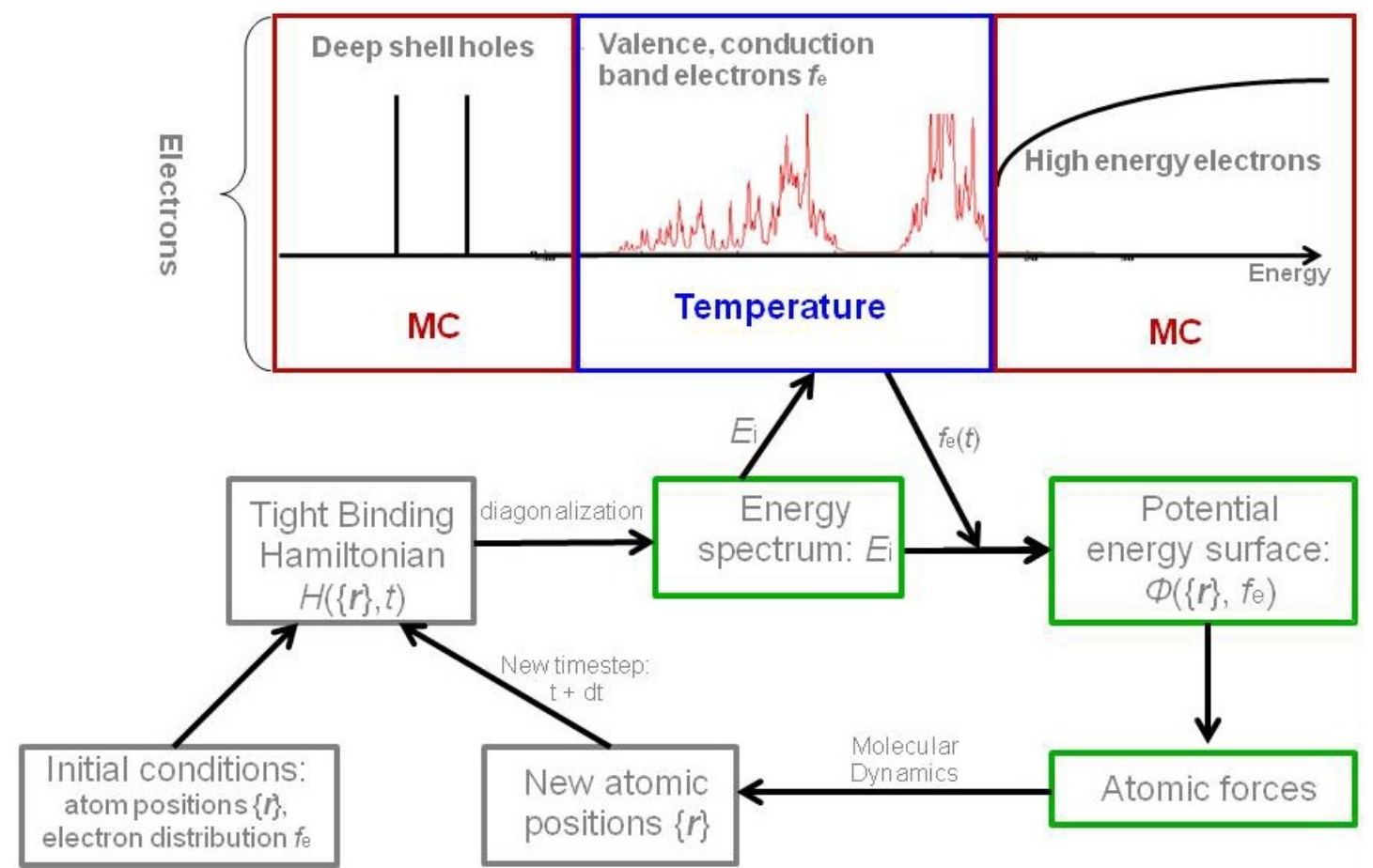

Figure 1. Schematic picture of the algorithm of the developed combined model consisting of the interconnected modules responsible for different calculations: Monte Carlo and Temperature equation for electrons, plus tight binding Molecular Dynamics for atomic motion. The figure is reproduced from [21].

The electron, which absorbed the photon, receives its energy and starts to perform secondary scatterings. We calculate the electron inelastic mean free path that depends on the current electron energy. The method of the generalized complex dielectric function is applied to obtain the cross-sections [28-32]. With this method, the differential cross-section of inelastic electron scattering $d \sigma\left(E_{e}\right) / d \omega$ is calculated from the complex dielectric function $\varepsilon(q, \omega)(\mathrm{CDF})$ within the first Born approximation as [28]:

$$
\frac{d \sigma}{d \omega}=\frac{\hbar}{\pi a_{0} E_{e}} \int_{q_{-}}^{q_{+}} \frac{1}{q} \operatorname{Im}\left(-\frac{1}{\varepsilon(q, \omega)}\right) d q
$$

where $E_{e}$ is the incident electron energy; integration is going over the current momentum within the limits $q_{ \pm}=\sqrt{2 m_{e}}\left(\sqrt{E_{e}} \pm \sqrt{E_{e}-\hbar \omega}\right) ; a_{0}$ is the Bohr radius; $q$ and $\omega$ are the transferred momentum and energy, respectively; $\hbar$ is the Planck constant. The total cross-section $\sigma$ can be obtained by the numerical integration of Eq.(1) for each electron energy $E_{e}$. Then, the mean free path, $l_{0}$ can be calculated, and the distance $l_{e}$ travelled by the electron can be sampled [33]:

$$
l_{e}=-\ln (\gamma) \cdot l_{0} ; l_{0}=\left(n_{e} \sigma\right)^{-1}
$$

using a random number $\gamma$ uniformly distributed in the interval $[0,1) ; n_{e}$ is the number of scattering centers (electrons). 
For calculation of the inelastic scattering cross-section one requires an appropriate model for the complex dielectric function. We employed Ritchie and Howie method of obtaining the complex dielectric function out of the optical data for different materials [28]. The dielectric function is then parametrized as a sum of Drude-type oscillators [28, 31, 32]:

$$
\operatorname{Im}\left[\frac{-1}{\varepsilon(\omega, q=0)}\right]=\sum_{i} \frac{A_{i} \gamma_{i} \hbar \omega}{\left(\hbar^{2} \omega^{2}-E_{0 i}^{2}\right)^{2}+\left(\gamma_{i} \hbar \omega\right)^{2}}
$$

where $E_{0 \mathrm{i}}$ is the characteristic energy of oscillator $i, A_{i}$ is the fraction of electrons which have energy $E_{0 \mathrm{i}}$, and $\gamma_{\mathrm{i}}$ is the $i$ th energy damping coefficient. The summation is running through all oscillators $n_{o s}$; this number $n_{o s}$ is defined by the particular shape of the experimentally measured imaginary part of the inverse of CDF. Fitting coefficients can be found following the algorithm described e.g. in [28, 31, 32].

We have constructed the CDF for the case of diamond and graphite, as limiting cases in our simulation. The corresponding coefficients are presented in Table I, the diamond case reproduces Ref. [21]. $n_{o s}$ equals to 6 for diamond and to 5 for graphite valence bands; the $\mathrm{K}$-shell is unaffected by the particular configuration of the carbon atoms (diamond vs graphite) and exhibits the same coefficients for both materials.

Table 1. Paramaterization of the complex dielectric function used to calculate electron inelastic cross-section, Eq. (3).

\begin{tabular}{|c|c|c|c|c|c|c|}
\hline & \multicolumn{3}{|c|}{ Diamond } & \multicolumn{3}{c|}{ Graphite } \\
\hline Shell & $\boldsymbol{E}_{\boldsymbol{0} \boldsymbol{i}}$ & $\boldsymbol{A}_{\boldsymbol{i}}$ & $\boldsymbol{\gamma}_{\boldsymbol{i}}$ & $\boldsymbol{E}_{\boldsymbol{0} \boldsymbol{i}}$ & $\boldsymbol{A}_{\boldsymbol{i}}$ & $\boldsymbol{\gamma}_{\boldsymbol{i}}$ \\
\hline Valence band & 22.3 & 17 & 2 & 10 & 8 & 2 \\
& 24.5 & 25 & 4 & 13 & 10 & 4 \\
& 29.2 & 185 & 5.5 & 19 & 115 & 5 \\
& 32 & 29 & 4 & 36 & 270 & 15 \\
& 35 & 221 & 11 & 47 & 515 & 38 \\
& 47 & 505 & 37 & & & \\
\hline K-shell & 250 & 480 & 200 & 250 & 480 & 200 \\
\hline
\end{tabular}

Using Eqs. (1-3) we can obtain the inelastic mean free path for an electron in diamond and graphite. Both are shown in Fig. 2. They are rather similar to each other, except that the graphite one is shifted to lower energies, because of the differences in the band gaps. The band gap of diamond is around $5.5 \mathrm{eV}$, while for graphite the band gap vanishes, as it is a semimetal. We apply this cross-section in our modeling of diamond, while neglecting the changes of the crosssection when the material turns into graphite, since such changes are not too large, and the number of high energy electrons is very small by the time of the phase transition (see Ref. [21]).

For the electron collisions, we determine the transferred energy out of the differential cross-section, Eq. (2) [33-35]. The corresponding energy transfer $\Delta E$ is determined by another random number $\gamma$ uniformly distributed in $[0,1)$ with the condition:

$$
\sigma \cdot \gamma=\int_{0}^{\Delta E} \frac{d \sigma}{d(\hbar \omega)} d(\hbar \omega)
$$

where one has to solve the equation (4) to obtain the transferred $\Delta E$ for each particular collision. This can be done numerically with the bisection method. 


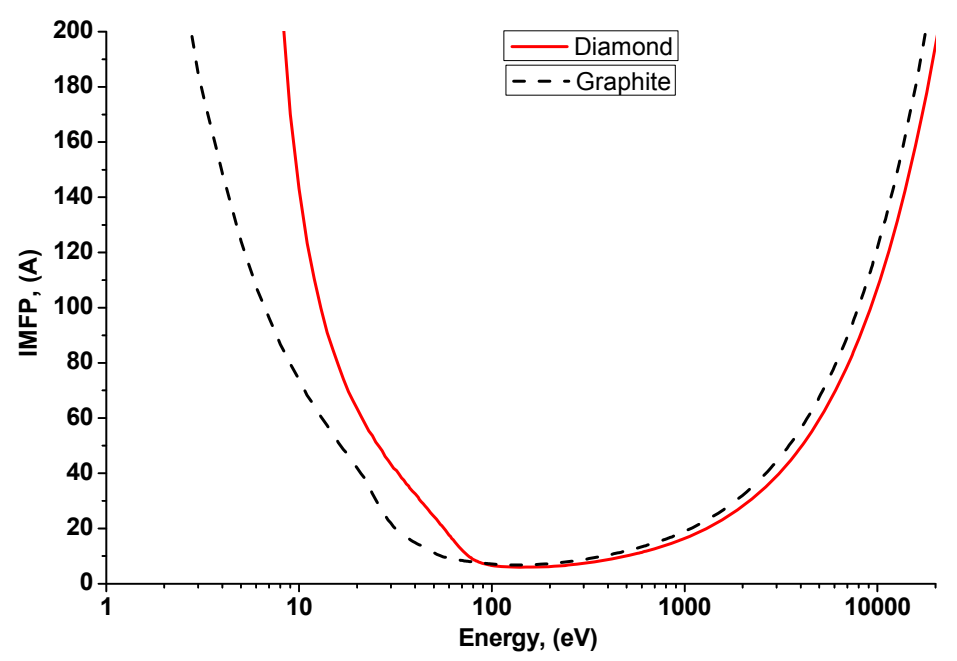

Figure 2. Inelastic mean free path of an electron in diamond and graphite obtained with the complex dielectric function formalism, Eqs. (1-3) and coefficients from Table I.

In case of secondary ionization, an electron is ionized if the transferred energy $\Delta E$, calculated with Eqs.(1) and (4) is higher than the ionization potential of the electron. Subsequent scatterings of secondary electrons produced are taken into account in the same manner. In case of Auger-decay of a K-shell hole, the hole is filled with the randomly chosen electron within the valence band, and another electron gains the excess energy [16-18].

We average the MC tracing of electrons over 10000 runs. The averaged quantities are then passed to the temperature equation as source terms defining the changes in the number of low energy electrons (as a mean number of excited and de-excited electrons) and their energy (which is brought in or out by interactions with high energy electrons). This procedure has to be repeated at each time-step of the simulation [21].

\subsection{Temperature of low energy electrons}

Since every change of an electronic state changes also the electron distribution function, we have to take this into account in our simulation. We do this under the assumption of partial equilibrium for the low energy electrons [21]. Their distribution function within the valence band and the low energy part of the conduction band is represented by the Fermi function which parameters, chemical potential $\mu$ and temperature $T_{e}$, are changing in time. These parameters enter later the calculation of interatomic forces. In our model, we follow the evolution of the number of electrons, which is defined by how many electrons are excited into high energy states or fall back to low energy states. This is defined within the MC step. Also, MC algorithm allows tracing the flows of energy between the two electron-energy domains and can be combined with other methods, see below [26]. The number of electrons $n_{e}$ and the energy of electrons $E_{e}$ are defined as $[21,26]$ :

$$
\begin{aligned}
& n_{e}=\sum_{E_{i}=E_{\min }}^{E_{\text {cut }}} 2\left(1+\exp \left(\left(E_{i}-\mu\right) / T_{e}\right)\right)^{-1}, \\
& E_{e}=\sum_{E_{i}=E_{\min }}^{E_{\text {cut }}} 2 E_{i}\left(1+\exp \left(\left(E_{i}-\mu\right) / T_{e}\right)\right)^{-1},
\end{aligned}
$$

where the summation is running over all energy states $E_{i}$ in the valence and the conduction bands. These energy levels are also evolving in time, as will be seen below; this effect originates from the atomic dynamics and forms the energy exchange between electrons and atoms. Thus, knowing the source terms from MC part, and the band-structure from the tight binding $\mathrm{MD}$, we can solve the inverse problem, Eq. (5), to determine the transient $\mu$ and $T_{e}$ on each time-step $[21,26]$. 


\subsection{Tight binding method}

Within the tight binding method, the atomic Hamiltonian consists of two parts: the attractive part, which depends on the electron configuration, and the repulsive part describing the repulsion of atomic cores [21, 22, 36, 37]. The diagonalization of this Hamiltonian is used to calculate the electronic energy levels at each time step, $E_{i}$ (band structure), entering Eqs.(5), and to determine the potential energy surface needed as the input to the equations of motion of atoms, as will be seen below. We used the transferrable tight binding method from Ref. [37], where the coefficients entering the Hamiltonian were fitted to $a b$-inito calculations. In this way, the hopping integrals are considered as fitting parameters to reproduce the band structure of the material. The method takes into account only the $s, p_{\mathrm{x}}, p_{\mathrm{y}}$ and $p_{\mathrm{z}}$ valence orbitals of carbon, which is sufficient to reproduce the band-structure with the required accuracy [36,37]. These parameters are functions of the atomic coordinates of all atoms in the simulation box. In this way, also the repulsive potential energy is fitted and parameterized. Properly chosen parameterization of the coefficients entering Hamiltonian allows this method to reproduce accurately both, diamond and graphite states [37].

All the corresponding coefficients and further details on the tight binging method applied to carbon are given in [21] and references therein. The potential energy surface can then be calculated as:

$$
\Phi\left(\left\{r_{i j}(t), t\right\}\right)=\sum_{E_{i}=E_{\min }}^{E_{c u t}} 2 E_{i}\left(1+\exp \left(\left(E_{i}-\mu\right) / T_{e}\right)\right)^{-1}+E_{\text {rep }}\left(\left\{r_{i j}\right\}\right)
$$

The transient electron distribution function, calculated in Eqs.(5), enters here. Thus, the time dependent electronic distribution directly affects the atomic motion.

\subsection{Molecular Dynamics method}

The classical molecular dynamics (MD) method $[38,39]$ is used to trace the atomic motion. The interatomic forces are calculated within the transferable tight binding method described in the previous section [36, 37]. The BornOppenheimer approximation used here relies on the assumption that fast moving electrons follow adiabatically the motion of ions. However, finite temperature for the electron distribution also allows including some nonadiabatic effects in an approximate way. So, we solve the classical equation of motion for all atoms within our simulation box, which are described by spatial coordinates and velocities. The collective potential energy surface, however, is calculated quantummechanically.

Accounting for the fact that the typical laser spot radius for the FLASH laser is $\sim 10 \mu \mathrm{m}$, and the X-ray photon penetration depth is above $100 \mathrm{~nm}$ [27], we choose only a small simulation box inside of the laser spot, and apply periodic boundary conditions. We analyzed two cases here: keeping constant volume of the simulation box, and keeping the constant pressure. We use the Parrinello-Rahman method which allows tracing the relaxing super-cell, which adjusts the volume for $P=$ const. Then, the equations of motion are written as [22, 40]:

$$
\begin{aligned}
& \ddot{\mathbf{s}}_{i}=-\partial \Phi\left(\left\{r_{i j}(t), t\right\}\right) / d s_{i}\left(g^{-1} / M_{a t}\right)-g^{-1} \dot{g} \dot{\mathbf{s}}_{i}, \\
& \ddot{h}_{\alpha \beta}=1 / M_{P R}\left(\sum_{i=1}^{N} M_{a t} \dot{\mathbf{s}}_{i}^{T}\left(h \dot{\mathbf{s}}_{i}\right)-P \partial \Omega / \partial h_{\alpha \beta}-\partial \Phi\left(\left\{r_{i j}(t), t\right\}\right) / \partial h_{\alpha \beta}\right),
\end{aligned}
$$

where $\boldsymbol{s}_{i}$ is the relative atomic coordinate within the super-cell; $h$ is the $(3 \times 3)$ matrix spanning the MD super-cell; $\Omega=\operatorname{det}(h)$ is the transient volume of the simulation box; $M_{a t}$ is the mass of an atom; $M_{P R}$ is the effective mass of the super-cell [21, 22]; and $g=h^{T} h$. Thus, simultaneous tracing of all the atoms, together with the changes of the super-cell itself, is performed for the case of $P=$ const. In case of $\mathrm{V}=$ const, the matrix $h$ is kept constant, and the second equation does not apply; thus, also the second term in the first equation of (7) vanishes, and the equation reduces to the standard Newtonian equation of motion written in the relative coordinates $\boldsymbol{s}_{i}$.

The potential energy surface $\Phi\left(\left\{r_{i j}\right\}\right)$ enters Eq. (7). It is defined by the transient electron distribution function, the positions of all the atoms in the super-cell, and by the transient electron band structure updated with the tight binding method at each time-step [21, 22]. We use Verlet algorithm in its velocity form for propagating the atomic coordinates and velocities in time, as well as for the supercell coordinates and velocities [38,39]. This ensures a stable numerical scheme. 


\section{RESULTS AND DISCUSSION}

We applied the model described above for femtosecond pulse irradiation of a solid diamond. The parameters were chosen to reproduce the typical experimental parameters of FLASH laser at DESY in Hamburg: $92 \mathrm{eV}$ photon energy, 10 fs as FWHM pulse duration. Fluence was adjusted so that the total absorbed dose is equal to $1 \mathrm{eV} /$ atom. This absorbed energy is above the graphitization threshold, which is reported to be $0.69 \mathrm{eV} /$ atom for $P=$ const case [21]. Here we also calculated the damage threshold for the $V=$ const case, which we found to be slightly higher: $0.74 \mathrm{eV} /$ atom.

Since for both cases in Fig. 2 the applied fluence is above the damage threshold, the phase transition can be expected. Indeed, for both of the applied MD modeling methods, $P=$ const and $V=$ const, one can observe an ultrafast phase transition. As it was presented in Ref. [21], the phase transition can be traced by the sudden strong fluctuation in the atoms' potential energy. Such occurring fluctuations are shown in Fig. 3.

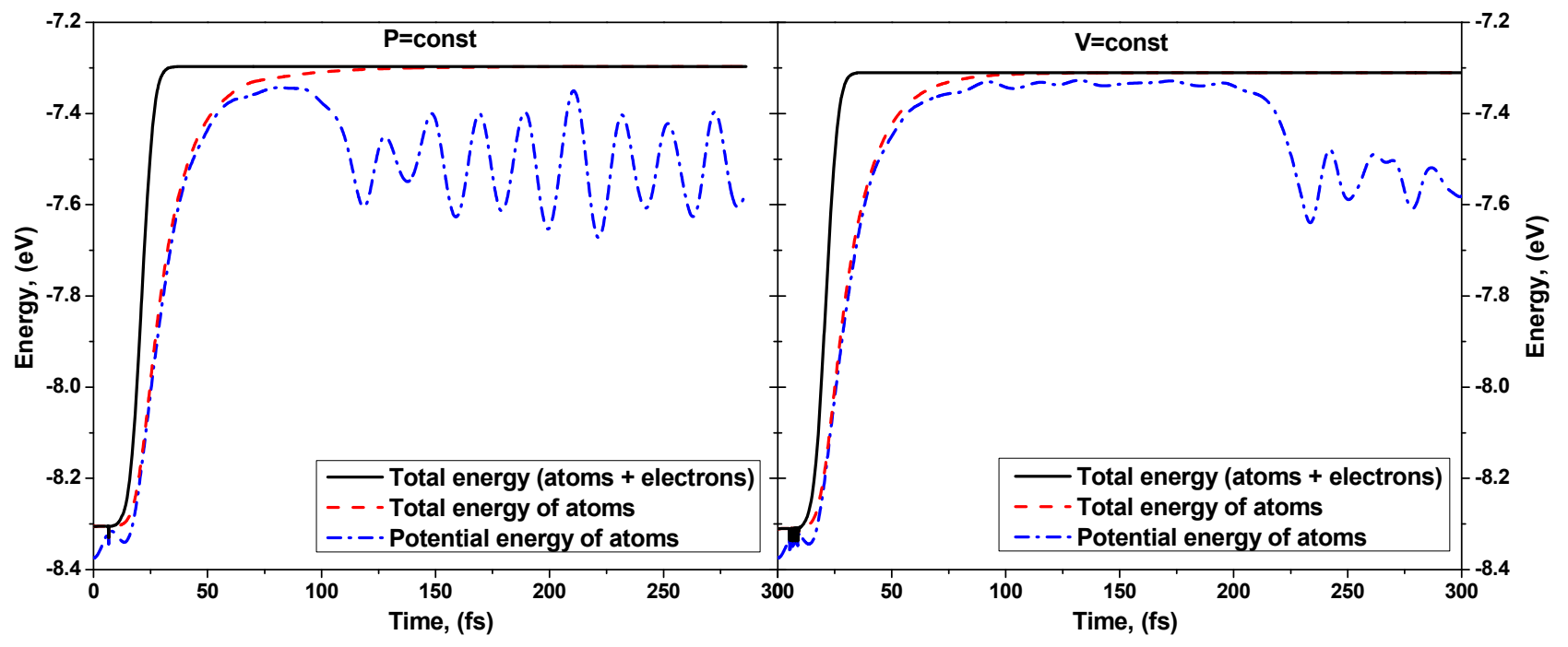

Figure 3. Energy distribution in diamond irradiated with $10 \mathrm{fs}$ laser pulse of $92 \mathrm{eV}$ photon energy and $1 \mathrm{eV} /$ atom absorbed energy. The total energy of the system (electrons and atoms) is a black solid line, the total energy of atoms is a red dashed line, and the potential energy of atoms is a blue dash-dotted line. The left panel shows the case of $P=$ const, while the right one is for $V=$ const.

Fig. 3 shows the transient energies in diamond after FLASH irradiation. The total energy (solid black line) is conserved within the numerical accuracy of the model, except for the times from $\sim 10$ to $\sim 30 \mathrm{fs}$, when the energy is delivered to the system by the pulse. At first, a part of the energy is stored in the high energy electrons, thus, the atomic energy (red dashed line) is lower than the total energy of the system. As the energy is transferred to the low energy domain, and thus becomes a part of the potential energy of atoms, the both energy curves meet. The potential energy of atoms (blue dashdotted line) is slightly fluctuating before and after the laser pulse, which represent the energy flows between the kinetic and potential energies of atoms. However, at the time of $\sim 90 \mathrm{fs}$, for the case of $P=$ const, the potential energy starts to oscillate much stronger. This is due to the fact that the atoms undergo phase transition into the new, graphite state [21]. In contrast to this phase transition on the sub-100 fs scales, for the constant volume case ( $V=$ const, right panel of Fig. 3 ), it takes longer time for the atomic system to realize its kinetic pathway to the new structural state: the phase transition occurs after $\sim 200 \mathrm{fs}$ after the pulse. When the atomic system is not relaxing as a whole, the super-cell is constrained with the fixed volume, than the phase transition takes longer. For lower fluencies, closer to the graphitization threshold, the delay of the phase transition is even longer, and can reach up to a picosecond timescales.

Fig. 4 presents the transient temperatures of electrons and atoms after the irradiation. The increasing oscillations of the potential energy of atoms, shown in Fig. 3, are directly reflected in the oscillations of the kinetic energy of atoms, and thus their temperature. The electron temperature is correspondingly decreasing on the same scales. Again, one can see that for the case of $V=$ const, right panel of Fig. 4, it takes some time for atoms to undergo the phase transition. After that, the electrons as well as atoms, have approximately the same temperatures for both cases, $P=$ const and $V=$ const. 


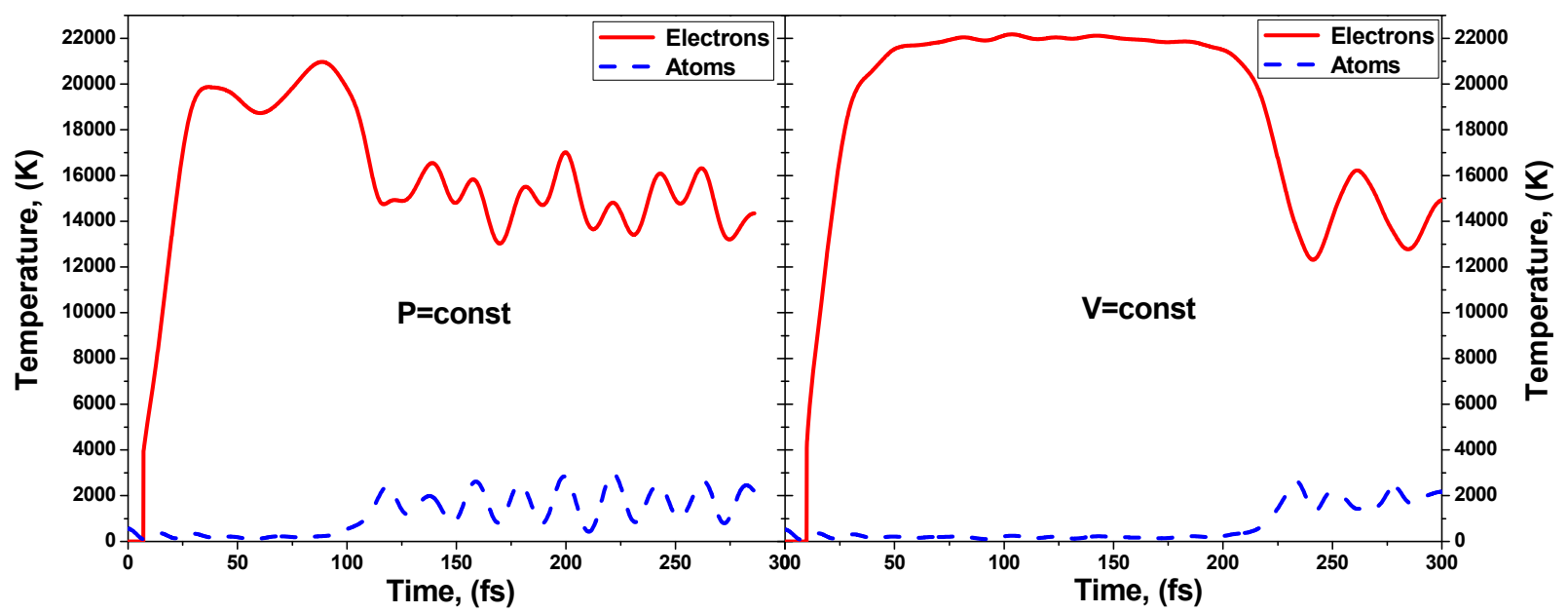

Figure 4. Temperatures of electrons (red solid line) and atoms (dashed blue line) in diamond irradiated with 10 fs laser pulse of $92 \mathrm{eV}$ photon energy and $1 \mathrm{eV} /$ atom absorbed energy. The left panel shows the case of $P=$ const, while the right one is for $V=$ const.

Time resolved experiments could clarify which approximation, $P=$ const vs $V=$ const, is more suitable for femtosecond Xray irradiation of semiconductors. The first results with the irradiation of graphite seem to indicate timescales of a few tens of femtosecond for amorphization, which corresponds more closely to the $P=$ const approximation [14]. More detailed experimental studies would help to clarify this issue.

\section{CONCLUSIONS}

In this contribution we presented our recently developed combined Monte-Carlo - Tight-Binding - Molecular Dynamics method. It allows incorporating in an adequate way the nonequilibrium electron kinetics on the changing band structure, together with the atomic dynamics on the time-evolving potential energy surface. Photoionization, electron secondary impact ionizations, thermalization of electron subsystem, changes of the band structure, atomic motion, and phase transitions are all addressed with this model. Application of the model to the case of diamond irradiated with FEL pulse (92 eV photon energy, $10 \mathrm{fs}$ FWHM) demonstrates the ultrafast phase transition of diamond to graphite phase. The timescale of this phase transition lays within few tens up to few hundreds of femtoseconds, depending on used approximations: the simulation with the constant pressure results in sub-100 fs phase transition, whereas constant volume leads to over $200 \mathrm{fs}$ timescales. The damage thresholds, calculated with both methods, are close to each other: 0.69 $\mathrm{eV} /$ atom vs $0.74 \mathrm{eV} /$ atom for $P=$ const and $V=$ const, respectively.

\section{ACKNOWLEDGMENTS}

The authors thank Prof. Dr. R. Santra (CFEL at DESY, Hamburg, Germany), Dr. D. Ivanov and V. Lipp (Universität Kassel, Germany) for fruitful discussions.

\section{REFERENCES}

[1] Rousse, A., Rischel, C., Fourmaux, S., Uschmann, I., Sebban, S., Grillon, G., Balcou, Ph., Förster, E., Geindre, J. P., Audebert, P., Gauthier, J. C., Hulin, D., "Non-thermal melting in semiconductors measured at femtosecond resolution," Nature 410, 65-68 (2001) 
[2] Sundaram, S. K., Mazur, E., "Inducing and probing nonthermal transitions in semiconductors using femtosecond laser pulses," Nature Mat. 1, 217-224 (2002)

[3] von der Linde, D., Sokolowski-Tinten, K., Bialkowski, J., "Laser-solid interaction in the femtosecond time regime." Appl. Surf. Sci. 109, 1-10 (1997)

[4] Cavalleri, A., Sokolowski-Tinten, K., Bialkowski, J., Schreiner, M., von der Linde, D., "Femtosecond melting and ablation of semiconductors studied with time of flight mass spectroscopy," J. Appl. Phys. 85, 3301(1999)

[5] Lee, R. W., Moon, S. J., Chung, H.-K., Rozmus, W., Baldis, H. A., Gregori, G., Cauble, R. C., Landen, O. L., Wark, J. S., Ng, A., Rose, S. J., Lewis, C. L., Riley, D., Gauthier, J.-C., Audebert, P., "Finite temperature dense matter studies on next-generation light sources," J. Opt. Soc. Am. B 20, 770-778 (2003)

[6] Gaudin, J. et al "Amorphous to crystalline phase transition in carbon induced by intense femtosecond x-ray freeelectron laser pulses," Phys. Rev. B 86, 024103 (2012)

[7] Emma, P. et al, "First lasing and operation of an Angstrom-wavelength free-electron laser." Nature Photonics 4, 641-647 (2010)

[8] Vinko, S. M. et al, "Creation and diagnosis of a solid-density plasma with an X-ray free-electron laser." Nature 482, 59-62 (2012)

[9] Zastrau, U. et al, "XUV spectroscopic characterization of warm dense aluminum plasmas generated by the freeelectron-laser FLASH," Laser and Particle Beams 30, 45-56 (2012)

[10] Nagler, B. et al, "Turning solid aluminium transparent by intense soft X-ray photoionization," Nature Physics 5, 693-696 (2009)

[11] Vinko, S. M. et al, "Electronic Structure of an XUV Photogenerated Solid-Density Aluminum Plasma," Phys. Rev. Lett. 104, 225001 (2010)

[12] Chalupsky, J. et al, "Damage of amorphous carbon induced by soft X-ray femtosecond pulses above and below the critical angle," Appl. Phys. Lett. 95, 031111 (2009)

[13] Gaudin, J. et al, "Photon energy dependence of graphitization threshold within diamond irradiated with an intense XUV FEL pulse," Phys. Rev. Lett. (2013, submitted)

[14] Hau-Riege, S. P, et al, "Ultrafast Transitions from Solid to Liquid and Plasma States of Graphite Induced by X-Ray Free-Electron Laser Pulses," Phys. Rev. Lett. 108, 217402 (2012)

[15] Fäustlin, R. R. et al, "Observation of Ultrafast Nonequilibrium Collective Dynamics in Warm Dense Hydrogen," Phys. Rev. Lett. 104, 125002 (2012)

[16] Ziaja, B., Medvedev, N., "Modelling ultrafast transitions within laser-irradiated solids," High En. Dens. Phys. 8, 1829 (2012)

[17] Medvedev, N., Zastrau, U., Förster, E., Gericke, D. O., Rethfeld, B., "Short-Time Electron Dynamics in Aluminum Excited by Femtosecond Extreme Ultraviolet Radiation," Phys. Rev. Lett. 107, 165003 (2011)

[18] Medvedev, N., Rethfeld, B., "Transient dynamics of the electronic subsystem of semiconductors irradiated with an ultrashort vacuum ultraviolet laser pulse," New J. Phys. 12, 073037 (2010)

[19] Ziaja, B., de Castro, A. R. B., Weckert, E., Möller, T., "Modelling dynamics of samples exposed to free-electronlaser radiation with Boltzmann equations," Eur. Phys. J. D 40, 465-480 (2006)

[20] Chapman, D. A., Gericke, D. O., "Analysis of Thomson Scattering from Nonequilibrium Plasmas," Phys. Rev. Lett. 107, 165004 (2011)

[21] Medvedev, N. Jeschke, H. O., Ziaja, B., "Nonthermal phase transitions in semiconductors induced by a femtosecond extreme ultraviolet laser pulse," New J. Phys. 15, 015016 (2013)

[22] Jeschke, H. O., Garcia, M. E., Bennemann, K. H., "Microscopic analysis of the femtosecond graphitization of diamond," Phys. Rev. B 60, R3701-R3704 (1999)

[23] Harmand, M., Coffee, R., Bionta, M. R., Chollet, M., French, D., Zhu, D., Fritz, D. M., Lemke, H. T., Medvedev, N., Ziaja, B., Toleikis, S., Cammarata, M., "Achieving few-femtosecond time-sorting at hard X-ray free-electron lasers," Nature Photonics 7, 215-218 (2013)

[24] Zijlstra, E. S., Walkenhorst, J., Garcia, M. E., "Anharmonic Noninertial Lattice Dynamics during Ultrafast Nonthermal Melting of InSb," Phys. Rev. Lett. 101, 135701 (2008)

[25] Lorazo, P., Lewis, L.J. and Meunier, M., "Thermodynamic pathways to melting, ablation, and solidification in absorbing solids under pulsed laser irradiation,"Phys. Rev. B 73, 134108-134130 (2006)

[26] Osmani, O., Medvedev, N., Schleberger, M., Rethfeld, B., "Energy dissipation in dielectrics after swift heavy-ion impact: A hybrid model," Phys. Rev. B 84, 214105 (2011)

[27] Henke, B. L., Gullikson, E. M., Davis, J. C., "X-Ray Interactions: Photoabsorption, Scattering, Transmission, and Reflection at $\mathrm{E}=50-30.000 \mathrm{eV}, \mathrm{Z}=1-92, "$ At. Data and Nucl. Data Tables 54, 181-342 (1993) 
[28] Ritchie, R. H., Howie, A., "Electron excitation and the optical potential in electron microscopy," Philos. Mag. 36, 463-481 (1977)

[29] Tanuma, S., Powell, C. J., Penn, D. R., "Calculations of Electron Inelastic Mean Free Paths II. Data for 27 Elements over the 50-2000eV Range," Surf. Interface Anal. 17, 911-926 (1991)

[30] Akkerman, A. F., Chernov, G. Ya., "Mean Free Paths by Inelastic Interactions, Stopping Powers, and Energy Straggling for Electrons of Energies up to $20 \mathrm{keV}$ in Various Solids," Phys. Stat. Sol. B 89, 329-333 (1978)

[31] Akkerman, A., Boutboul, T., Breskin, A., Chechik, R., Gibrekhterman, A., Lifshitz, Y., "Inelastic Electron Interactions in the Energy Range $50 \mathrm{eV}$ to $10 \mathrm{keV}$ in Insulators: Alkali Halides and Metal Oxides," Phys. Stat. Sol. B 198, 769-784 (1996)

[32] Medvedev, N., "Modeling ultrafast electronic processes in solids excited by femtosecond VUV-XUV laser Pulse," AIP Conf. Proc. 1464, 582 (2012)

[33] Medvedev, N. A., Rethfeld, B., "Electron kinetics in semiconductors and metals irradiated with VUV-XUV femtosecond laser pulses," Proc. SPIE 8077, 80770Q (2011)

[34] Eckstein, W. "Computer Simulations of Ion-Solid Interactions," Springer-Verlag, New York, (1991)

[35] Jacoboni, C., Reggiani, L., "The Monte Carlo method for the solution of charge transport in semiconductors with applications to covalent materials," Rev. Mod. Phys. 55, 645 (1983)

[36] Jeschke, H. O., "Theory for Optically Created Nonequilibrium in Covalent Solids", PhD Thesis: Freie Universitaet Berlin, (2000)

[37] Xu, C. H., Wang, C. Z., Ting, C. S., Su, W. P., "A transferable tight-binding potential for carbon," J. Phys. Condens. Matter 4, 6047-6054 (1992)

[38] Allen, M. P., Tildesley, D. J., "Computer Simulation of Liquids," Oxford University Press, Oxford (1987)

[39] Rapaport, D. C., "The Art of Molecular Dynamics Simulation," Cambridge University Press, Cambridge (2002)

[40] Parrinello, M., Rahman, A., "Crystal Structure and Pair Potentials: A Molecular-Dynamics Study," Phys. Rev. Lett. 45, 1196-1199 (1980) 